\title{
Missing checkerboards? An absence of competitive signal in Alnus-associated ectomycorrhizal fungal communities
}

A number of recent studies suggest that interspecific competition plays a key role in determining the structure of ectomycorrhizal (ECM) fungal communities. Despite this growing consensus, there has been limited study of ECM fungal community dynamics in abiotically stressful environments, which are often dominated by positive rather than antagonistic interactions. In this study, we examined the ECM fungal communities associated with the host genus Alnus, which live in soils high in both nitrate and acidity. The nature of ECM fungal species interactions (i.e. antagonistic, neutral, or positive) was assessed using taxon co-occurrence and DNA sequence abundance correlational analyses. ECM fungal communities were sampled from root tips or mesh in-growth bags in three monodominant $A$. rubra plots at a site in Oregon, USA and identified using Illumina-based amplification of the ITS1 gene region. We found a total of 175 ECM fungal taxa; 16 of which were closely related to known Alnus-associated ECM fungi. Contrary to previous studies of ECM fungal communities, taxon co-occurrence analyses on both the total and Alnusassociated ECM datasets indicated that the ECM fungal communities in this system were not structured by interspecific competition. Instead, the co-occurrence patterns were consistent with either random assembly or significant positive interactions. Pair-wise correlational analyses were also more consistent with neutral or positive interactions. Taken together, our results suggest that interspecific competition does not appear to determine the structure of all ECM fungal communities and that abiotic conditions may be important in determining the specific type of interaction occurring among ECM fungi. 
1 Missing checkerboards? An absence of competitive signal in Alnus-associated ectomycorrhizal

5 Biology, Lewis \& Clark College, Portland, OR, 3. Department of Biology, Stanford University, 
7 Corresponding Author: Peter Kennedy, 1445 Gortner Ave., St. Paul, MN, USA. Email:

8 kennedyp@umn.edu, Phone: 612-624-8519, Fax: 612-625-1738.Introduction

9 A common way to assess the role of interspecific competition or facilitation in

10 determining community structure is experimental manipulation involving the removal of

11 neighboring individuals. This approach has been widely used in ecological studies examining

12 biotic determinants of plant and animal communities (Connell 1983; Schoener 1983), but

13 carrying out similar manipulations in field-based studies of soil microbial communities is less

14 feasible due to the inability to selectively manipulate species-level neighborhood composition. A

15 widely proposed alternative is to look at species distribution patterns, with Diamond's (1975)

16 study of bird distributions in the New Guinea archipelago being one of most well-recognized

17 examples. In that study, the presence of certain bird species on a given island was associated with

18 the absence of other species (and vice versa on other islands), resulting in a series of 'forbidden

19 species combinations' or 'checkerboard distributions', which were posited to be the result of

20 competitive exclusion (Diamond 1975). This technique provided an important step forward in

21 assessing the role of species interactions in field-based studies at the community level, but it has

22 been frequently noted that analyses of species co-occurrence patterns need to include

23 comparisons with patterns generated from communities assembled randomly to maximize

24 inference (Connor \& Simberloff 1979; Gotelli \& Graves 1996).

25 Since the 1970 s, species co-occurrence analyses have been used to assess the possibility

26 of species interactions in a wide range of organisms, including both macro- and microorganisms

27 (Gotelli \& McCabe 2002; Horner-Devine et al., 2006). Plant-associated fungal communities,

28 which have diverse ecological roles in ecosystems (Smith \& Read 2008; Rodriguez et al., 2009),

29 have shown a full range of co-occurrence patterns, including those consistent with both positive

30 and antagonistic interactions (Koide et al., 2005; Pan \& May 2009; Gorzelak et al., 2012;

31 Ovaskainen et al., 2010; Pickles et al., 2012; Toju et al., 2013). For ectomycorrhizal (ECM) 
32 fungi, the dominant microbial eukaryotes in many temperate and some tropical forest soils (Smith

33 and Read 2008), these analyses have consistently found evidence of less species co-occurrence

34 than expected by chance (Koide et al., 2005; Pickles et al., 2010; Pickles et al., 2012). This

35 suggests that competitive interactions may play a significant role in structuring the communities

36 of this fungal guild (Kennedy 2010). The initial studies of species co-occurrence patterns in ECM

37 fungal communities looked only in forests dominated by conifer hosts, but a recent study in

38 Fagus sylvatica forests in Europe also found evidence of significantly lower than expected co-

39 occurrence patterns (Wubet et al., 2012). This latter result indicates that the predominance of

40 antagonistic interactions in determining ECM fungal community structure may be a common,

41 host-lineage independent phenomenon. Importantly, however, other ecological and evolutionary

42 factors aside from species interactions can also be responsible for non-random species co-

43 occurrence patterns (Gotelli \& McCabe 2002; Ovaskainen et al. 2010), so caution must be 44 applied in inferring underlying mechanisms.

45 In this study, we focused on assessing the community co-occurrence distributions of ECM

46 fungi associated with the host genus Alnus. Unlike other ECM host genera with large

47 geographical distributions, the ECM fungal communities associated with Alnus trees have been

48 consistently found to be both species poor and highly host specific (Tedersoo et al., 2009;

49 Kennedy \& Hill 2010; Kennedy et al., 2011; Bogar \& Kennedy 2013; Põlme et al., 2013; Roy et

50 al., 2013). The mechanisms driving this atypical structure have long been thought to be related to

51 the co-presence of nitrogen-fixing Frankia bacteria, which can have strong biotic and abiotic

52 effects on Alnus-associated ECM fungal communities (Walker et al., 2014). In particular, the high

53 rates of nitrification present in Alnus forest soils (due to the high inputs and decomposition of

54 nitrogen-rich leaf litter) results in significantly higher nitrate and acidity levels than those present

55 in most other ECM-dominated forest soils (Daniere et al. 1986, Miller et al., 1992; Martin et al.,

56 2003; Walker et al., 2014). Elevated levels of both of these abiotic factors have been shown to 
57 inhibit the growth of many ECM fungi (Hung and Trappe 1983; Lilleskov et al., 2002) and, using

58 an experimental pure culture approach, Huggins et al., (in press) recently demonstrated that

59 Alnus-associated ECM fungi have a greater ability to tolerate high nitrate and acidity conditions

60 compared to non-Alnus-associated ECM fungi.

61 Given the ability of Alnus-associated ECM fungi to grow in conditions that are generally

62 considered abiotically stressful, we hypothesized that ECM fungal species co-occurrence patterns

63 in Alnus forests may differ from those present in forests dominated by other ECM hosts.

64 Specifically, we speculated that competitive interactions would be less prevalent in this study

65 system, based on the fact that many studies of vascular plants have shown that the nature of

66 species interactions often changes from antagonistic to positive with increasing levels of abiotic

67 stress (Bertness \& Callaway 1994, Gómez-Aparicio et al., 2004, but see Michalet et al., 2006). To

68 examine this hypothesis, we examined the co-occurrence patterns of the ECM fungal

69 communities present in three mono-dominant plots of Alnus rubra in the western United States.

70 ECM fungal communities were sampled on root tips and in soil. For the latter, we used sand-

71 filled mesh in-growth bags, which allow for efficient, well-replicated community sampling of

72 fungal hyphae growing in soil (Wallander et al., 2001, Branco et al., 2013). To identify the ECM

73 fungi present in the study, we used high throughput Illumina sequencing, which has been

74 increasingly used to profile ECM fungal community composition (McGuire et al., 2013, Smith \& 75 Peay 2014).

\section{Materials \& Methods}

\section{Study Location}

78 The study site was located on the eastern side of the Coast Range mountains in 79 northwestern Oregon, U.S.A. (latitude: N $45.820 \mathrm{~W}$ 123.05376, elevation: $462 \mathrm{~m}$ ). Temperatures 80 at the site are moderate (mean annual temperature $=8.7^{\circ} \mathrm{C}, \min =-1.2^{\circ} \mathrm{C}, \max =23.8^{\circ} \mathrm{C}$ ), with 
81 significant precipitation between October and May followed by drier summer months (total $=$

$821742 \mathrm{~mm}$ ). The specific study location is part of a long-term research project examining the

83 effects of different forest management practices on A. rubra growth (see the Hardwood

84 Silvicultural Cooperative (HSC) website for details, http://www.cof.orst.edu/coops/hsc). The

85 HSC site used, Scappoose (HSC 3209), was established in 1995. Prior to the implementation of

86 the HSC work, the site was a second-growth coniferous forest, which was clear-cut and replanted

87 with a series of monodominant A. rubra plots. A. rubra seedlings were planted from nursery

88 stock (Brooks Tree Farm, Brooks, OR) during the beginning of their second year of growth.

89 Seedling ECM status at the time of planting was not assessed (Frankia nodules were noted to be

90 absent), but nursery fumigation practices indicate colonization was unlikely (Brooks Nursery,

91 pers. com.).

92 Our experiment was conducted in three $1600 \mathrm{~m}^{2}$ plots at HSC 3209. The plots, which

93 were located approximately $100 \mathrm{~m}$ apart, differed in initial A. rubra stem density (Plot $2=628$,

94 Plot $4=1557$, and Plot $8=3559$ stems/ha), but had no other forest management practices

95 applied. Despite the differences in stem density, A. rubra fine root density did not differ

96 significantly among the three plots (Fig. S1). The understories in all three plots were colonized by

97 arbsucular mycorrhizal plants (dominated by Mahonia nervosa and Claytonia perfoliata), with no

98 other ECM hosts besides A. rubra present. Soils were classified as well-drained Tolamy loams

99 (USDA Soil Survey, Columbia County, OR). Within each plot, we located a 9 x 9 m subplot and

100 overlaid a 100 point grid, with each point being separated by $1 \mathrm{~m}$. We chose this subplot size to

101 avoid any dead stems in the canopy immediately above the sampling area, while at the same time

102 maximizing the number of samples taken per subplot. At each point in Plot 4, which was sampled

103 for ECM root tips, a $5 \mathrm{~cm}$ diameter $\mathrm{x} 10 \mathrm{~cm}$ deep soil core was taken on May 31, 2013. In Plots

1042 and 8, which were sampled for ECM communities present in soil, a 5 x $5 \mathrm{~cm}$ mesh bag was 
105 buried at each point $5 \mathrm{~cm}$ below the soil surface. The bags were made of anti-static polyester

106 fabric with 300 micron diameter pores. This pore size allowed fungal hyphae to grow into the

107 bags, but prevented penetration of plant roots. We filled the bags with twice autoclaved \#3 grade

108 Monterey aquarium sand (Cemex, Marina, CA, USA). Aluminum tags on fluorescent string were

109 added to facilitate bag recovery. The mesh bags at Plot 2 were buried on February 1, 2013 and

110 February 22 at Plot 8 . They were left undisturbed in the soil until May 31, when all were

111 harvested. After removal from the soil, we placed the mesh bags into individual plastic bags and

112 then onto ice for transport back to the laboratory. Soil cores and bags were stored at $4{ }^{\circ} \mathrm{C}$ for $<96$

113 hours before further processing.

114 Molecular Analyses

115 We processed the root tip samples by gently washing all roots away from the soil and

116 removing all ECM colonized root tips from each core under a 10X dissecting scope ( 10-50 root

117 tips/core). All roots from each core were extracted using individual MoBio PowerSoil kits

118 (Hercules, CA, USA), following manufacturer's instructions for maximum DNA yields. For the

119 mesh bags, we followed the protocol outlined in Branco et al., (2013), which provided a cheaper

120 and quicker protocol compared to direct DNA extraction from the sand within the mesh bags.

121 Briefly, each bag (including a negative control that was taken to the field, but not buried) was

122 emptied into a sterile $50 \mathrm{ml}$ centrifuge tube. We added $10 \mathrm{ml}$ of sterile deionized water and

123 vortexed each tube for two minutes, followed by a five minute settling period (hyphae have been

124 previously observed to float to the water surface). We then transferred the top two ml top of

125 water to a new $2 \mathrm{ml}$ centrifuge tube and contents were pelleted via centrifugation. On the same

126 day, we extracted total genomic DNA form the pellets using the Sigma REDExtract-N-Amp kit

127 (Sigma-Aldrich, St, Louis, MO, USA) following manufacturer's instructions. Root tips and

128 extracts were stored for one week at $-20^{\circ} \mathrm{C}$ prior to PCR amplification. 
130 extractions (three cores contained no roots) into a single template for PCR. In contrast, for each

131 mesh bag sample as well as extraction controls, we conducted individual PCR reactions. We

132 processed these two types of samples differently because we were primarily interested in the

133 spatial co-occurrence patterns in the soil ECM fungal communities and therefore only used the

134 root tip samples to create a local sequence reference set of known Alnus-associated ECM taxa

135 against which the mesh bag data could be compared. For all PCR reactions, we used the barcoded

136 ITS1F and ITS2 primer set of Smith \& Peay (2014), with each sample run in triplicate and pooled

137 to minimize heterogeneity. Successful PCR products were determined by gel electrophoresis and

138 magnetically cleaned using the Agencourt AMPure XP kit (Beckman Coulter, Brea, CA, USA)

139 according to manufacturer's instructions. Final product concentrations were quantified using a

140 Qubit dsDNA HS Fluorometer (Life Technologies, Carlsbad, CA, USA). Root tip and bag

141 samples were run at different sequencing facilities under the same general conditions. For the

142 root tips, the single PCR product was run at the University of Minnesota Genomics Center using

$143250 \mathrm{bp}$ paired-end sequencing on the MiSeq Illumina platform. For the bags, we pooled the 192

144 successfully amplified bag samples at equimolar concentration and ran them on the same

145 platform at the Stanford Functional Genomics Facility using 250 bp paired-end sequencing on the

146 MiSeq Illumina platform. A spike of $20 \%$ and $30 \%$ PhiX was added to the runs to achieve

147 sufficient sample heterogeneity, respectively. Raw sequence data and associated metadata from

148 both the root tip and bag samples were deposited at MG-RAST (http://metagenomics.anl.gov/)

149 under project \#1080.

150 Bioinformatic Analyses

151 We used the software packages QIIME (Caporaso et al., 2010) and MOTHUR (Schloss et 152 al., 2009) to process the sample sequences. Raw sequences were demultiplexed, quality filtered 
153 using Phred $=20$, trimmed to 178 base pairs, and ends were paired, followed by filtering out of

154 sequences that had any ambiguous bases or a homopolymer run of $9 \mathrm{bp}$. Following the guidelines

155 discussed in Nguyen et al., (in press), we employed a multi-step operational taxonomic unit

156 (OTU) picking strategy by first clustering with reference USEARCH (including de novo chimera

157 checking) at $97 \%$ sequence similarity, followed by UCLUST at $97 \%$ sequence similarity. We

158 used a 97\% similarity threshold because it the most commonly employed in community-level

159 ECM fungal studies, although some lineages, including Alnicola, may have greater sequence

160 similarity among species (Tedersoo et al. 2009, Rochet et al. 2011). To assess the validity of the

$16197 \%$ threshold for sequences based on only ITS1 versus the full ITS region (i.e. ITS1, 5.8S, and

162 ITS2), we examined seven known Alnus-associated Tomentella taxa (i.e. those present in

163 Kennedy et al., 2011) and found that that threshold resulted in the same number of OTUs in both

164 cases (data not shown). The UNITE database (Kõljalg et al., 2013) was used in both chimera

165 checking and OTU clustering, with singleton OTUs discarded to minimize the effects of

166 artifactual sequences (Tedersoo et al. 2010b). We assigned taxonomic data to each OTU with

167 NCBI BLAST+ v2.2.29 (Altschul et al., 1990), using a custom fungal ITS database containing

168 the curated UNITE SH database (v6) (http://unite.ut.ee/repository.php, Kõljalg et al., 2013) and

169 more than 600 vouchered fungal specimens, including 46 representative sequences from Alnus

170 forests at other HSC locations in Oregon (Kennedy \& Hill 2010) and Mexico (Kennedy et al.

171 2011). Since sequences that had low subject length:query length matches were typically non-

172 fungal, we further filtered out sequences with matches $\leq 90 \%$ to BLAST (i.e. at least $90 \%$ of the

173 bases in the input sequence matches to another sequence in the database at some identity level).

174 Using the remaining sequence dataset, we rarefied all samples to 12946 sequences, which

175 was the lowest number of sequences obtained across the 192 samples. Since there has recently

176 been a question raised about the validity of rarefaction in next generation sequencing analyses

177 (McMurdie and Holmes 2014), we also analyzed the data without rarefaction. We obtained very 
178 similar results (Table S1), so present the data based on rarefied samples only. ECM OTUs within

179 each sample were parsed out using a python script that searches for genera names from a list of 180189 known ECM genera and their synonyms (Branco et al., 2013, appended from Tedersoo et al.,

181 2010a). While this script provides a strong general filter for sorting the data by fungal lifestyle,

182 some taxa belonging to clades that are polyphyletic for the ECM habit (e.g. Lyophyllum,

183 Sebacinales) as well as taxa with low matches to Genbank (e.g. Uncultured Fungus) can be of

184 questionable trophic status. For each of these groups, we carefully checked both the sequence 185 matches and placement of our OTUs within phylogenetic trees of the clades to determine whether

186 these taxa were properly classified at ECM. The resulting sample x OTU matrix contained 190

187 ECM taxa represented by at least one sequence per sample $(\min =1$, median $=34$, mean $=1334$,

$188 \max =209,187)$. We found that 15 of the 190 OTUs present were highly similar $(>97 \%$ similar $)$

189 to ECM fungi present in the dipterocarp rainforests of Malaysia, which were concurrently being

190 studied in the Peay lab using the same next-generation sequencing approach (Fig. 1). Because

191 these OTUs represented accidental contamination probably during library construction, they were

192 eliminated from the final analyses. Although an additional 80 OTUs had greater than $>97 \%$

193 similarity to taxa found in the Borneo study, because their closest BLAST match was not from

194 Borneo, we conservatively considered these taxa as having cosmopolitan distributions and

195 included them in the final analyses. The final OTU $\times$ sample matrix, including taxonomic

196 matches and representative of sequences for each OTU, can be found in Table S2.

197

\section{Statistical Analyses}

Taxon co-occurrence patterns of the ECM fungal communities present in bag samples were assessed using the program EcoSim (Gotelli \& Entsminger 2009), with presence-absence matrices for Plots 2 and 8 being analyzed separately. (The root data from Plot 4 could not be analyzed for sample-level co-occurrence due to the pooled sequencing approach for those 


\section{PeerJ Reviewing Manuscript}

202 samples). We utilized the C-score algorithm (Stone \& Roberts 1990), which compares the

203 number of checkerboard units (i.e. 1,0 x 0,1$)$ between all pairs of species in the observed matrix

204 ( $\left.\mathrm{C}_{\text {observed }}\right)$ to that based in random permutations of the same matrix $\left(\mathrm{C}_{\text {expected }}\right.$, i.e. the null models).

205 Since randomized permutations of a matrix can be achieved in multiple ways (see Gotelli \&

206 Entsminger 2009 for details), we analyzed our datasets using both the 'fixed-fixed' and 'fixed-

207 equiprobable' options (which are recommended by the program guide and used in the previous

208 ECM fungal co-occurrence analyses). In both options, the row (i.e. taxon) totals were fixed, so

209 that the total abundances of each taxon in the observed and null matrices were identical. In the

210 'fixed-equiprobable' option, however, the column (i.e. sample) totals in the null matrices were no

211 longer equivalent to those in the observed matrix. Instead, all samples in the null matrices had an

212 equal probability of being colonized by any of the taxa in the observed matrix, which effectively

213 eliminates differences in taxon richness among samples.

214 Of the ECM taxa present in the final root tip and bag datasets, over $90 \%(167 / 183)$

215 belonged to species never previously encountered with Alnus (Table S2, AlnusMatch $=$ No).

216 Unlike other ECM host systems with large geographic ranges, the ECM fungal community

217 associated with Alnus hosts is remarkably well characterized at local (Tedersoo et al., 2009,

218 Kennedy et al., 2010, Walker et al., 2014), regional (Kennedy et al., 2011, Roy et al., 2013, and

219 global scales (Polme et al., 2013). As such, it is highly likely the majority of the novel OTUs

220 encountered were not part of the active ECM community in our plots, but rather present simply

221 either as spores or additional lab contaminants. To account for this issue, we divided our

222 checkerboard analyses into five different input matrices for the bag dataset (Plots 2 and 8 ). The

223 first matrix included all 183 ECM fungal taxa (referred to as "All"). The second matrix included

224 the 16 taxa that had $>97 \%$ similarity matches to ECM samples from Alnus forests (referred to as

225 Alnus). The third matrix included only the 8 taxa that were encountered on ECM root tips in Plot 
2264 (referred to as AlnusRootOnly). To assess the robustness of the results generated using the

227 larger Alnus matrix, the fourth matrix excluded the three most frequent and abundant species

228 (Tomentella3, Alnicola1, Tomentella2) (referred to as AlnusMinusTop3). Finally, the fifth matrix

229 included just the 10 taxa in the genus Tomentella (from the larger Alnus matrix) to look for

230 evidence of species interactions among this subset of closely related taxa (referred to as

231 AlnusTomentellaOnly). For all of the aforementioned C-score analyses, taxa present in less than 5

232 bag samples were removed, as low frequency taxa are generally considered non-informative

233 (Koide et al., 2005). The observed input matrices were compared to 5000 null matrices.

234 Significant differences between the observed matrix C-score and that of the null matrices were

235 determined along with standardized effect sizes (SES). Observed C-scores significantly higher

236 than those generated from the null matrices are consistent with a community being structured by

237 competitive interactions, whereas $\mathrm{C}_{\text {observed }}$ significantly lower than the $\mathrm{C}_{\text {expected }}$ is consistent with

238 positive interactions.

To further assess the degree of association between known Alnus ECM fungal taxa, we

240 also used an abundance-based approach (as opposed to the co-occurrence analyses, which are

241 based on binary presence/absence data). Specifically, we calculated the pair-wise Spearman rank

242 correlation coefficients among all pairs of the 16 Alnus-associated taxa using the cor function in

243 R (R Development Core Team 2013). Coefficients $>0.30$ were tested for significance with the

244 cor.test function. To account for multiple tests $(\mathrm{n}=13)$, we used a Bonferroni-corrected $P$ value of

2450.003 . With the same data set, we also tested for the presence of spatial autocorrelation using the

246 mgram function in the ECODIST package in R. We first converted the sequence abundance

247 datasets in both Plots 2 and 8 into dissimilarity matrices using the Bray-Curtis Index and then

248 compared those to a Euclidean distance matrix of sampling points for each plot. For the Mantel 
correlogram tests, we used the n.class $=0$ option, which uses Sturge's equation to determine the appropriate number of distance classes.

\section{Results}

We found 183 total ECM fungal taxa in the study (Table S2); 16 of which matched closely

253 to known Alnus-associated ECM fungi. In the mesh bags, Alnus-associated ECM fungal taxa represented six of the ten most abundant OTUs present, including the dominant ECM fungal taxon, Tomentella3, which was present in all the bag samples in both plots and had sequence abundances nearly ten-fold higher than any other taxon (Fig. 2a,b). Two other Alnus-associated taxa, Alnicola 1 and Tomentella2, were also present in all samples, whereas the remaining Alnusassociated ECM fungal taxa had frequencies varying from 2-96\% (Plot 2 mean $=25 \%$, Plot 8 mean $=31 \%$ ) and lower sequence abundances. Eight of the 16 Alnus-associated ECM fungal taxa were present on both roots and in the bags, with abundances that were very similar (Fig. 1a). Of the eight ECM fungal taxa found on root tips, all were previously encountered on $A$. rubra root tips at other sites in Oregon, while the eight taxa found exclusively in bags had not been previously documented (Kennedy \& Hill 2010).

(1)

ECM fungal taxon co-occurrence patterns were largely consistent between plots, but different between null models. Of the ten tests (i.e. 5 matrix types $x 2$ plots) using the 'fixedfixed' permutation option, nine indicated that the observed ECM fungal community did not differ significantly from random assembly (Table 1). In one case, Plot 2 All, the observed ECM fungal community had significantly more co-occurrence than expected by chance. In contrast, in the ten tests using the 'fixed-equiprobable' permutation option, three indicated that the observed ECM fungal community did not differ significantly from random assembly, while seven found that the 
271 observed ECM fungal community had significantly more co-occurrence than expected by chance.

272 Results remained the same for Alnus ECM fungal communities whether the top three taxa were

273 removed or not. The Alnus and AlnusRootOnly analyses did differ under the 'fixed-equiprobable'

274 option, with the former showing greater than expected co-occurrence and the latter having a

275 pattern no different than one based on random assembly. Additionally, in the

276 AlnusTomentellaOnly analysis, the ECM fungal community showed greater than expected co-

277 occurrence in Plot 2 but not in Plot 8. In all of these cases, significant antagonistic patterns were

278 not observed.

Spearman rank analyses revealed that pair-wise sequence abundances of some of the 16

280 Alnus ECM fungal taxa were significantly positively correlated (Table 2). The specific significant

281 combinations varied between plots, with only taxon pair (Alnicola1 \& Tomentella9) showing

282 significant positive correlations in both plots. Although a number of pair-wise correlations had

283 negative values (suggesting negative rather than positive interactions), none of them were

284 significant, even when considered at a $P$ value of 0.05 . In addition, the Mantel correlogram

285 analyses found no clear evidence of spatial autocorrelation in the Alnus-associated ECM fungal

286 communities. In Plot 2, there was no significant autocorrelation at any distance, while in Plot 8

287 there was a single significant positive correlation between samples located 1-2 $\mathrm{m}$ apart (Fig. S2, 288 S3).

289 Discussion

290 We found that the ECM fungal communities in A. rubra forests displayed a different 291 pattern of taxon co-occurrence compared to those seen for other ECM fungi. Unlike the 292 consistent previous findings of less co-occurrence among species than expected by chance (Koide 
293 et al., 2005; Pickles et al., 2012; Wadet et al., 2012), we observed no evidence of spatial patterns

294 consistent with interspecific competition in Alnus-associated ECM fungal communities. In

295 contrast, we consistently found co-occurrence patterns that were either no different from random

296 assembly or consistent with positive interactions. Although we did not measure soil nitrate and

297 acidity conditions in this study (see Martin et al., (2003) and Walker et al., (2014) for values from

298 comparable age A. rubra forests at other sites in Oregon), Alnus soils are consistently

299 characterized by abiotic conditions are generally considered stressful to ECM fungi. The results

300 we obtained are thus consistent with the 'stress gradient hypothesis', which posits that species

301 interactions shift from negative to positive as environmental conditions become harsher (Bertness

302 \& Callaway 1994). Although we emphasize that the patterns we found in this study are based

303 solely on correlative inference, there is some experimental evidence that may support the stress

304 gradient hypothesis for ECM fungal community dynamics. Koide et al., (2005) found a shift from

305 significant negative co-occurrence patterns in their control plots to non-significant co-occurrence

306 patterns in plots where either tannins or nitrogen were added experimentally. While they did not

307 explicitly analyze these manipulations in terms of stress, both increased tannin and nitrogen

308 levels have been shown to inhibit the growth of multiple ECM taxa (Koide et al., 1998; Cox et

309 al., 2010). The direction of the response in the Koide et al., (2005) study is consistent with greater

310 abiotic stress resulting in a decrease in antagonistic ECM fungal interactions. At the same time, it

311 is plausible that resource limitation was eliminated with the addition of nitrogen, which could

312 have allowed for greater spatial co-existence among ECM fungi. Since the Alnus system has

313 naturally higher nitrogen availability than most ECM forests due to the co-presence of nitrogen-

314 fixing Frankia bacteria, it is also possible that greater resource abundance could drive the co-

315 occurrence patterns we observed. Given the fact that the pattern could be explained by either

316 increasing stress or resource availability, additional experimental tests are needed to distinguish

317 among these explanations. One promising approach would be to examine the taxon co-occurrence 
318 patterns in younger and older Alnus forests, since soil nitrate and acidity concentrations increase

319 in these forests over time (Daniere et al. 1986, Martin et al., 2003). If the stress gradient

320 hypothesis were the most plausible explanation, then we would expect to see competitive and

321 facilitative interactions to be dominant, respectively.

The presence of co-occurrence patterns consistent with significant negative species

323 interactions was also missing in our analysis of more closely related ECM fungal taxa. For the ten

324 Alnus-associated members of the genus Tomentella, co-occurrence patterns either did not differ

325 significantly from random assembly or reflected an effect of positive interactions. Like the larger

326 community analyses, this result also differs from previous experimental studies, where strong

327 antagonistic interactions among closely related ECM fungal taxa have been observed (Kennedy

328 2010). In a similarly designed study that also assessed ECM fungi with taxon co-occurrence

329 analyses, Pickles et al., (2012) found patterns consistent with strong interspecific competition

330 among a suite of Cortinarius species in a Scottish Pinus sylvestris forest. Although it has long

331 been assumed that competition may be stronger in more closely related species due to greater

332 overlap in resource utilization, a meta-analysis by Cahill et al., (2008) found little consistent

333 evidence to support this supposition. Mayfield \& Levine (2010) further questioned the validity of

334 phylogenetic relatedness as a good proxy for competitive strength by showing that in certain

335 abiotic environments competition may actually select for more closely related taxa than expected

336 by chance (i.e. phylogenetic clustering). The Alnus ECM system is particularly interesting in this

337 respect because while the fungal communities associated with Alnus hosts are both species poor

338 and highly host specific, they include taxa from a number of distantly related lineages (Rochet et

339 al., 2011). Although explanations for this higher-level phylogenetic patterning are still lacking,

340 our current results suggest that competitive processes among both closely and more distantly 
341 related taxa are not a key factor generating the atypical structure of Alnus ECM fungal 342 communities.

343 Some positive spatial associations have been observed in other studies of ECM fungal 344 communities (Agerer et al., 2002; Koide et al., 2005; Pickles et al., 2012), and have been

345 suggested to be due to complementary resource acquisition abilities of among individual taxa

346 (Jones et al., 2010). We speculate that in Alnus forests positive associations among ECM fungi

347 could also reflect possible amelioration of local abiotic conditions. Huggins et al., (in press)

348 found that Alnus-associated ECM fungi could more effectively buffer changes in local $\mathrm{pH}$

349 environments than non-Alnus ECM fungi, which may be key to persistence in the high acidity

350 soils present in Alnus forests. While the exact buffering mechanism is not yet known, if it

351 involves the release of molecules into the external environment, growing directly adjacent to

352 another ECM fungus may result in greater buffering of local $\mathrm{pH}$ conditions than when growing in

353 isolation. We believe it is important to note, however, that the patterning of positive associations

354 were patchy and not consistent between plots, so it is hard to determine if local $\mathrm{pH}$ buffering is

355 actually significant without local measurements of $\mathrm{pH}$ for each sample. Furthermore, sequence

356 abundance of individual taxa has been shown not to correlate linearly with initial fungal tissue or

357 DNA abundance in other studies using NGS techniques (Amend et al., 2010, Nguyen et al.,

358 2014), so caution must be applied in using sequence abundance as an accurate ecological proxy.

359 Like the co-occurrence and correlation-based patterns, we found that spatial

360 autocorrelation patterns observed in Alnus ECM fungal communities were also anomalous

361 relative to other studies. The specific distance of spatial autocorrelation appears to vary among

362 systems, but there is typically strong spatial autocorrelation among community samples located

363 less than $5 \mathrm{~m}$ apart (e.g. Lilleskov et al. 2004, Bahram et al. 2013). While the spatial extent of

364 our study was very limited (the most distant samples within plots were only $\sim 12 \mathrm{~m}$ apart), the 
365 absence of spatial signal was not surprising based on previous studies of Alnus ECM fungal

366 communities. Both Pritsch et al. (2010) and Kennedy et al. (2011) found individual Alnus ECM

367 fungal taxa that were almost identical genetically (at least in the ITS region) in forests located

368 thousands of kilometers apart and, in a global scale analysis, Polme et al. (2013) found many

369 Alnus ECM OTUs were distributed across geographically distant samples. Theoretically, the

370 absence of dispersal limitation should make the detection of non-random distribution patterns

371 more likely if biotic interactions (either negative or positive) are strong determinants of

372 community structure. The classic work of Diamond (1975) is a good example, as the bird

373 populations studied across the New Guinea archipelago were not dispersal limited, yet exhibited

374 many checkerboard distribution patterns. As such, we do not think the atypical nature of the taxon

375 co-occurrence patterns in Alnus ECM fungal communities that we observed were driven by the

376 also atypical spatial correlation patterns.

As the results observed in this study differed in multiple ways from those found

378 previously, we had some concern they were caused by an artifact of our identification or

379 sampling methodology. Unlike previous examinations of taxon co-occurrence for ECM fungi, we

380 used next-generation sequencing (NGS) to identify the communities present. NGS methods

381 provide much greater sequencing depth per sample (Smith and Peay 2014), which may have

382 allowed us to more effectively document the ECM fungal communities present in each sample

383 compared to previous studies. We found that the three most abundant Alnus-associated ECM

384 fungi were present in every bag sample in both plots, which has not been observed in other

385 systems. Although the presence of spatially ubiquitous taxa will result in a lower total number of

386 checkerboard units observed (as 1,0 is possible but not 0,1 ), it has the same effect on both the

387 observed and null matrices and therefore should not bias statistical comparisons of $\mathrm{C}_{\text {observed }}$ versus

$388 \mathrm{C}_{\text {expected. }}$ We checked this by eliminating the three ECM fungal taxa present in every sample and 
389 found functionally identical results to those when those taxa were included (Table 2). A second 390 difference between this and related studies was the sampling of ECM fungal hyphal communities

391 in mesh bags. Previous studies assessing co-occurrence patterns have largely focused on ECM

392 root tips, but Koide et al., (2005) found very similar taxon co-occurrence patterns for root-tip and

393 soil-based analyses of ECM fungal communities in the same Pinus resinosa forest. Based on that

394 result, and the fact that the sequence abundances of all the ECM fungi present on $A$. rubra root

395 tips and the mesh bags showed highly similar patterns (Table S2), we do not believe assessing

396 ECM hyphal communities was the source of our incongruous results either (however, in

397 hindsight, a better experimental design would have been to sample the mesh bags and the ECM

398 root tips directly around them within each plot). A third difference is the restricted taxonomic

399 richness of Alnus ECM fungal communities. This explanation, however, also seems unwarranted,

400 as Pickles et al., (2012) showed highly significant negative co-occurrence patterns in matrices of

401 equivalent sizes. It is also possible that variation in soil nutrient availability could drive Alnus

402 ECM fungal community structure and, because it was relatively homogenous in our small-sized

403 plots, the resulting taxon distribution patterns were largely random. While we reiterate that we

404 did not directly measure soil nutrient availability in this study, other studies of Alnus ECM fungi

405 have shown some significant correlations between community structure and soil organic matter

406 and nutrients such as K and Ca (Becerra et al., 2005, Tedersoo et al., 2009, Roy et al., 2013,

407 Polme et al., 2013; see Richard (1968) for a possible mechanism). In those studies, however, the

408 percent of variance explained by soil nutrients was generally low, so we believe it is unlikely that

409 variation in resource availability was the primary determinant of the distribution patterns

410 observed. We recognize that additional differences likely exist, but feel confident that the co-

411 occurrence results we observed are ecologically accurate and not generated by methodological or

412 sampling artifact. 
NGS techniques clearly represent a powerful and efficient way to assess the richness and

414 dynamics of fungal communities (Smith \& Peay 2014), but we found that additional data quality

415 control analyses beyond the standard sequence quality thresholds and chimera checking were

416 needed to properly characterize ECM fungal community composition. Specifically, we found that

417 a relatively high number of ECM fungal taxa present that appeared to be the result of PCR

418 contamination. The PCR reactions of our extraction and PCR controls produced no bands

419 indicating positive product, but the sensitivity of NGS techniques and the Illumina platform in

420 particular makes the amplification of single DNA molecules highly probable (Tedersoo et al.,

421 2010b; Peay et al., 2013). Fortunately, the atypical and well-described nature of Alnus ECM

422 fungal communities made it relatively easy to identify the most obvious non-Alnus associated

423 taxa and remove them prior to the final analyses. For taxa that belonged to ECM fungal lineages

424 known to associate with Alnus hosts but which had not been previously documented, it was more

425 difficult to determine their status (i.e. whether they represented PCR contaminants, were present

426 in A. rubra soils as spores, or actually colonizing A. rubra root tips). In particular, the status of

427 Thelephoraceae1, which had the third highest sequence abundance in the full dataset, was

428 interesting because the closest BLAST match to Thelephoraceae1 was an ECM fungal root tip

429 sample from Betula occidentalis in British Columbia, Canada. Bogar \& Kennedy (2013) found

430 that ECM fungal communities present on Alnus and Betula hosts can overlap, so it is possible this

431 taxon was overlooked in previous surveys of Alnus ECM fungal communities that used less

432 sensitive methods. However, the absence of this taxon from any the root tip samples in Plot 4

433 suggests that it was most likely present simply as spores rather than an active member of the

434 Alnus-associated ECM fungal community. Despite the unclear status of this taxon as well as

435 many others with lower abundance, the co-occurrence patterns showed the same general results

436 whether taxa of unknown status were included or not, suggesting the overall results were robust.

437 In less well-characterized ECM fungal and other microbial systems, however, the potential for 
438 inclusion of spurious taxa is sufficiently high that we strongly recommend the sequencing of

439 negative extraction and PCR controls to help try to account for any lab-based contamination

440 (Nguyen et al., in press).

441 Taken together, our results suggest that while many ECM fungal communities appear to

442 be strongly affected by competitive interactions, those present in Alnus forests are not. Although

443 the reasons for this difference are not fully resolved in this study, the possibility of greater abiotic

444 stress changing the way in which species interact in Alnus forests is likely an important factor.

445 The application of ecological theories such as the stress gradient hypothesis to better understand

446 the factors driving ECM fungal community structure has grown rapidly in recent years (Peay et

447 al., 2008; Koide et al., 2014) and new technologies such as next generation sequencing continue

448 to make the study of ECM fungi increasingly tractable for ecologists. While we welcome this

449 synergy, we stress the importance of a solid foundation in fungal biology as well as critical

450 awareness of the limitations of molecular-based identification techniques to successfully integrate

451 ECM fungi into the ecological mainstream.

\section{Acknowledgements}

We thank A. Bluhm and D. Hibbs for assistance with using the HSC study location, L.

454 Bogar, V. Engebretson, J. Huggins, P. King for assistance with experiment implementation and 455 harvest, J. Walker for assistance with DNA extractions, D. Smith for assistance with NGS 456 processing, and members of the Peay Lab, C. Fernandez, R. Koide, M. Gardes and one 457 anonymous reviewer for critical comments on a previous version of this manuscript. 


\section{References}

Agerer R, Grote R, Raidl S. 2002. The new method "micromapping”, a means to study speciesspecific associations and exclusions of ectomycorrhizae. Mycological Progress 1:155-166.

Altschul SF, Gish W, Miller W, Myers WE, Lipman DJ. 1990. Basic local alignment search tool. Journal of Molecular Biology 215:403-410.

Amend AS, Seifert KA, Bruns TD. 2010. Quantifying microbial communities with 454 pyrosequencing: does read abundance count? Molecular Ecology 19:5555-5565.

Bahram M, Kõljalg U, Courty PE, Diédhiou AG, Kjøller R, Põlme S, Ryberg M, Veldre V, Tedersoo L. 2013. The distance decay of similarity in communities of ectomycorrhizal fungi in different ecosystems and scales. Journal of Ecology 101:1335-1344

Becerra A, Zak MR, Horton TR, Micolini J. 2005. Ectomycorrhizal and arbuscular mycorrhizal colonization of Alnus acuminata from Calilegua National Park (Argentina). Mycorrhiza $15: 525-531$.

Bertness MD, Callaway R. 1994. Positive interactions in communities. Trends in Ecology \& Evolution 9:191-193.

Bogar LM, Kennedy PG. 2013. New wrinkles in an old paradigm: neighborhood effects can modify the structure and specificity of Alnus-associated ectomycorrhizal fungal communities. FEMS Microbiology Ecology 83:767-77.

Branco S, Bruns TD, Singleton I. 2013. Fungi at a small scale: spatial zonation of fungal assemblages around single trees. PloS ONE 8:e78295. 
478 Cahill JF, Kembel SW, Lamb EG, Keddy PA. 2008. Does phylogenetic relatedness influence the 479 strength of competition among vascular plants? Perspectives in Plant Ecology, Evolution $480 \quad$ and Systematics $10: 41-50$.

481 Caporaso JG, Kuczynski J, Stombaugh J, Bittinger K, Bushman FD, Costello EK, Fierer N, Peña 482 AG, Goodrich JK, Gordon JI, Huttley GA, Kelley ST, Knights D, Koenig JE, Ley RE, 483 Lozupone CA, McDonald D, Muegge BD, Pirrung M, Reeder J, Sevinsky JR, Turnbaugh PJ, 484 Walters WA, Widmann J, Yatsunenko T, Zaneveld J, Knight R. 2010. QIIME allows analysis 485 of high-throughput community sequencing data. Nature Methods 7:335-336.

486 Connell JH. 1983. On the prevalence and relative importance of interspecific competition: 487 evidence from field experiments. American Naturalist 122:661-696.

488 Connor EF, Simberloff D. 1979. The assembly of species communities - chance or competition. $489 \quad$ Ecology 60:1132-1140.

490 Cox F, Barsoum N, Lilleskov E, Bidartondo M. 2010. Nitrogen availability is a primary 491 determinant of conifer mycorrhizas across complex environmental gradients. Ecology $492 \quad$ Letters 13:1103-1113.

Danière C, Capellano A, Moiroud A. 1986. Nitrogen transfer in a natural stand of Alnus incana L. Moench. Acta Oecologica/Oecologia Plantarum 7: 165-175 evolution of communities. Harvard University Press, Cambridge. 342-444. 
497 Gómez-Aparicio L, Zamora R, Gómez JM, Hódar JA, Castro J, Baraza E. 2004. Applying plant

498 facilitation to forest restoration: a meta-analysis of the use of shrubs at nurse plants.

$499 \quad$ Ecological Applications 14: 1128-1138.

500 Gorzelak MA, Hambleton S, Massicotte HB. 2012. Community structure of ericoid mycorrhizas

501 and root-associated fungi of Vaccinium membranaceum across an elevation gradient in the

502 Canadian Rocky Mountains. Fungal Ecology 5:36-45.

503 Gotelli NJ, Entsminger GL. 2009. EcoSim: Null models software for ecology. Version

$504 \quad 7:$ Available at: http://garyentsminger.com/ecosim.htm.

505 Gotelli NJ, Graves GR. 1996. Null models in ecology. Smithsonian Institution Press,

$506 \quad$ Washington, DC.

507 Gotelli NJ, McCabe DJ. 2002. Species co-occurrence: a meta-analysis of J. M. Diamond's

$508 \quad$ assembly rules model. Ecology 83:2091-2096.

509 Horner-Devine MC, Silver JM, Leibold MA, Bohannan BJM, Colwell RK, Fuhrman JA, Green

510 JL, Kuske CR, Martiny JBH, Muyzer G, Ovreås L, Reysenbach AL, Smith VH. 2007. A

511 comparison of taxon co-occurrence patterns for macro- and microorganisms. Ecology

$512 \quad 88: 1345-1353$.

513 Huggins JL, Talbot JM, Gardes M, Kennedy PG. In Press. Unlocking the environmental keys to

514 host specificity: differential nitrate and acidity tolerance by Alnus-associated

515 ectomycorrhizal fungi. Fungal Ecology. DOI: http://dx.doi.org/10.1016/

$516 \quad$ j.funeco.2014.04.003 
517 Hung L, Trappe J. 1983. Growth variation between and within species of ectomycorrhizal fungi

518 in response to $\mathrm{pH}$ in vitro growth. Mycologia 75:234-241.

519 Jones MD, Twieg BD, Ward V, Barker J, Durall DM, Simard SW. 2010. Functional

520 complementarity of Douglas-fir ectomycorrhizas for extracellular enzyme activity after

$521 \quad$ wildfire or clearcut logging. Functional Ecology 24:1139-1151.

522 Kennedy PG. 2010. Ectomycorrhizal fungi and interspecific competition: species interactions,

523 community structure, coexistence mechanisms, and future research directions. New

$524 \quad$ Phytologist 187:895-910.

525 Kennedy PG, Garibay-Orijel R, Higgins LH, Angeles-Arguiz R. 2011. Ectomycorrhizal fungi in

526 Mexican Alnus forests support the host co-migration hypothesis and continental-scale

527 patterns in phylogeography. Mycorrhiza 21:559-568.

528 Kennedy PG, Hill LT. 2010. A molecular and phylogenetic analysis of the structure and

529 specificity of Alnus rubra ectomycorrhizal assemblages. Fungal Ecology 3:195-204.

530 Kjoller R. 2006. Disproportionate abundance between ectomycorrhizal root tips and their

$531 \quad$ associated mycelia. FEMS Microbiology Ecology 58:214-224.

532 Koide R, Fernandez CW, Malcolm G. 2014. Determining place and process: functional traits of 533 ectomycorrhizal fungi that affect both community structure and ecosystem function. New

$534 \quad$ Phytologist 201:433-439.

535 Koide R, Suomi L, Stevens C, McCormick L. 1998. Interactions between needles of Pinus

536 resinosa and ectomycorrhizal fungi. New Phytologist 140:539-547. 
537 Koide RT, Xu B, Sharda J, Lekberg Y, Ostiguy N. 2005. Evidence of species interactions within $538 \quad$ an ectomycorrhizal fungal community. New Phytologist 165:305-316.

539 Kõljalg U, Nilsson RH, Abarenkov K, Tedersoo L, Taylor AFS, Bahram M, Bates ST, Bruns TD,

540 Bengtsson-Palme J, Callaghan TM, Douglas B, Drenkhan T, Eberhardt U, Dueñas M,

541 Grebenc T, Griffith GW, Hartmann M, Kirk PM, Kohout P, Larsson E, Lindahl BD, Lücking

542 R, Martín MR, Matheny PB, Nguyen NH, Niskanen T, Oja J, Peay KG, Peintner U, Peterson

543 M, Põldmaa K, Saag L, Saar I, Schüßler A, Scott JA, Senés C, Smith ME, Suija A, Taylor

544 DL, Telleria MT, Weiss M, Larsson KH. 2013. Towards a unified paradigm for sequence-

545 based identification of fungi. Molecular Ecology 22:5271-5277.

546 Lilleskov EA, Fahey TJ, Horton TR, Lovett GM. 2002. Belowground ectomycorrhizal fungal

547 community change over a nitrogen deposition gradient in Alaska. Ecology 83:104-115.

548 Lilleskov EA, Bruns TD, Horton TR, Taylor DL, Grogan P. 2004. Detection of forest stand-level

549 spatial structure in ectomycorrhizal fungal communities. FEMS Microbiology Ecology

$550 \quad 49: 319-332$.

551 Martin KJ, Posavatz NJ, Myrold DD. 2003. Nodulation potential of soils from red alder stands

552 covering a wide age range. Plant and Soil 254:187-192.

553 Mayfield MM, Levine JM. 2010. Opposing effects of competitive exclusion on the phylogenetic $554 \quad$ structure of communities. Ecology Letters 13:1085-1093.

555 McGuire, KL, Payne SG, Palmer MI, Gillikin CM, Keefe D, Kim SJ, Gedallovich SM, Discenza $556 \underline{\mathrm{J}}, \underline{\text { Rangamannar R}}, \underline{\text { Koshner JA, }}$ Massmann AL, Orazi G, Essene A, Leff JW, Fierer N

557 Digging the New York City Skyline: Soil Fungal Communities in Green Roofs and City $558 \quad$ Parks. PLoS ONE 8: e58020. 
559 McMurdie PJ, Holmes S. 2014. Waste not, want not: why rarefying microbiome data is

$560 \quad$ inadmissable. PloS Computional Biology 10: e1003531.

561 Michalet R, Brooker RW, Cavieres LA, Kikvidze Z, Lortie CJ, Pugnaire FI, et al. 2006. Do biotic

562 interactions shape both sides of the humped-back model of species richness in plant

563 communities? Ecology Letters 9:767-73.

564 Nguyen NH, Smith D, Peay KG, Kennedy PG. In press. Parsing ecological signal from noise in 565 next generation amplicon sequencing. in press. New Phytologist DOI: 10.1111/nph.12923

566 Ovaskainen O, Hottola J, Siitonen J. 2010. Modeling species co-occurrence by multivariate 567 logistic regression generates new hypotheses on fungal interactions. Ecology 91:2514$568 \quad 2521$.

569 Pan JJ, May G. 2009. Fungal-fungal associations affect the assembly of endophyte communities 570 in maize (Zea mays). Microbial Ecology 58:668-78.

571 Peay KG, Baraloto C, Fine PVA. 2013. Strong coupling of plant and fungal community structure 572 across western Amazonian rainforests. The ISME Journal 7:1852-61.

573 Peay KG, Kennedy PG, Bruns TD. 2008. Fungal community ecology: a hybrid beast with a 574 molecular master. Bioscience 58:799-810.

575 Pickles BJ, Genney DR, Potts JM, Lennon JJ, Anderson IA, Alexander IJ. 2010. Spatial and 576 temporal ecology of Scots pine ectomycorrhizas. New Phytologist 186:755-768. 
577 Pickles BJ, Genney DR, Anderson IA, Alexander IJ. 2012. Spatial analysis of ectomycorrhizal

578 fungi reveals that root tip communities are structured by competitive interactions. Molecular

$579 \quad$ Ecology 21:5110-5123.

580 Põlme S, Bahram M, Yamanaka T, Nara K, Dai YC, Grebenc T, Kraigher H, Toivonen M, Wang

581 PH, Matsuda Y, Naadel T, Kennedy PG, Kõljalg U, Tedersoo L. 2013. Biogeography of

582 ectomycorrhizal fungi associated with alders (Alnus spp.) in relation to biotic and abiotic

$583 \quad$ variables at the global scale. New Phytologist 198:1239-49.

584 Pritsch K, Becerra A, Polme S, Tedersoo L, Schloter M, Agerer R. 2010. Description and

585 identification of Alnus acuminata ectomycorrhizae from Argentinean alder stands.

$586 \quad$ Mycologia 102:1263-1273.

587 Richard L. 1968. Ecologie de l'aune vert (Alnus viridis Chaix). Documents pour la carte de la $588 \quad$ végétation des Alpes 6: 107-147.

589 Rochet J, Moreau PA, Manzi S, Gardes M. 2011. Comparative phylogenies and host

590 specialization in the alder ectomycorrhizal fungi Alnicola, Alpova and Lactarius

591 (Basidiomycota) in Europe. BMC Evolutionary Biology 11:40.

592 Rodriguez RJ, White JF, Arnold AE, Redman RS. 2009. Fungal endophytes: diversity and 593 functional roles. New Phytologist 182:314-330.

594 Roy, M, Rochet J, Manzi S, Jargeat P, Gryta H, Moreau PA, Gardes M. 2013. What determines

595 Alnus-associated ectomycorrhizal community diversity and specificity? A comparison of

596 host and habitat effects at a regional scale. New Phytologist 198:1228-1238. 
597 Schloss PD, Westcott SL, Ryabin T, Hall JR, Hartmann M, Hollister EB, Lesniewski RA, Oakley

598 BB, Parks DH, Robinson CJ, Sahl JW, Stres B, Thallinger GG, Van Horn DJ, Weber CF.

599 2009. Introducing Mothur: Open-Source, Platform-Independent, Community-Supported

600 Software for Describing and Comparing Microbial Communities. Applied and

$601 \quad$ Environmental Microbiology 75:7537-7541.

602 Schoener TW. 1983. Field experiments on interspecific competition. American Naturalist

$603 \quad 122: 240-285$.

604 Smith DP, Peay KG. 2014. Sequence depth, not PCR replication, improves ecological inference 605 from next generation DNA sequencing. PloS ONE 9:e90234.

606 Smith SE, Read DJ. 2008. Mycorrhizal Symbiosis. 3rd edition. Elsevier, New York.

607 Stachowicz JJ. 2001. Mutualism, facilitation, and the structure of ecological communities.

$608 \quad$ BioScience 51:235-246.

609 Stone L, Roberts A. 1990. The checkerboard score and species distributions. Oecologia 85:74-

$610 \quad 79$.

611 Tedersoo L, May TW, Smith ME. 2010a. Ectomycorrhizal lifestyle in fungi: global diversity,

612 distribution, and evolution of phylogenetic lineages. Mycorrhiza 20:217-263.

613 Tedersoo L, Nilsson RH, Abarenkov K, Jairus T, Sadam A, Saar I, et al. 2010b. 454

614 Pyrosequencing and Sanger sequencing of tropical mycorrhizal fungi provide similar results

615 but reveal substantial methodological biases. New Phytologist 188:291-301. 
616 Tedersoo L, Suvi T, Jairus T, Ostonen I, Põlme S. 2009. Revisiting ectomycorrhizal fungi of the

617 genus Alnus: differential host specificity, diversity and determinants of the fungal

618 community. New Phytologist 182:727-735.

619 Toju H, Yamamoto S, Sato H, Tanabe AS. 2013. Sharing of diverse mycorrhizal and root-

620 endophytic fungi among plant species in a oak-dominated cool-temperate forest. PloS ONE

$621 \quad 8: \mathrm{e} 78248$.

622 Walker JKM, Cohen H, Higgins LM, Kennedy PG. 2014. Testing the link between community

623 structure and function for ectomycorrhizal fungi involved in a global tri-partite symbiosis.

$624 \quad$ New Phytologist 202:287-296.

625 Wallander H, Nilsson LO, Hagerberg D, Baath E. 2001. Estimation of the biomass and seasonal

626 growth of external mycelium of ectomycorrhizal fungi in the field. New Phytologist

$627 \quad 151: 753-760$.

628 Wubet T, Christ S, Schöning I, Boch S, Gawlich M, Schnabel B, Fischer M, Buscot F. 2012.

629 Differences in soil fungal communities between European beech (Fagus sylvatica L.)

630 dominated forests are related to soil and understory vegetation. PloS ONE 7:e47500. 


\section{Figure 1}

Rank-abundance plot of all 190 (inset) and top 20 ectomycorrhizal (ECM) fungal taxa sampled in this study.

The top 20 ECM fungal taxa are color coded by whether there are known to be associated with Alnus hosts (black), of unknown host origin (grey), or laboratory contaminants (white).

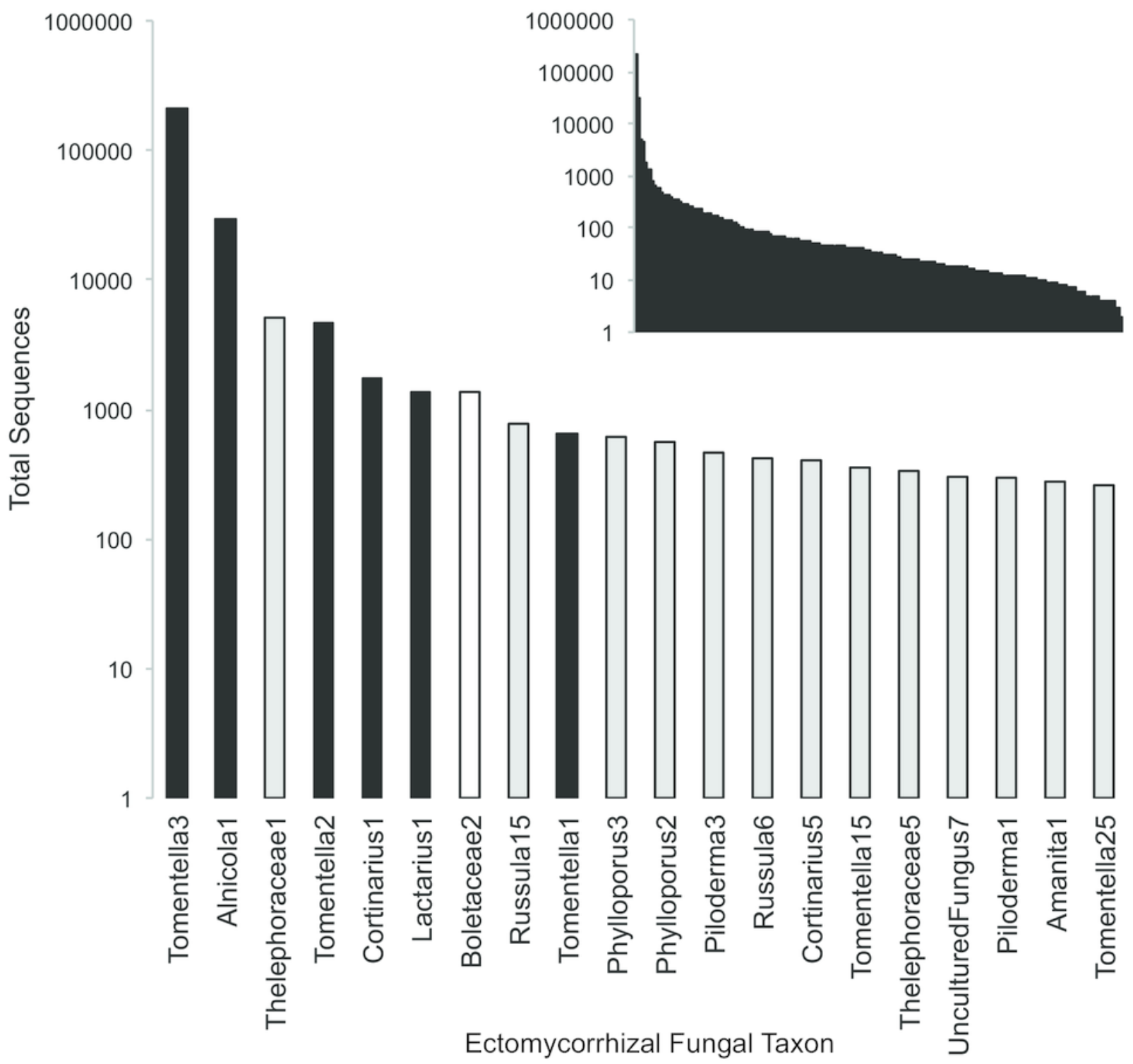


Figure 2

Rank-abundance (A) and rank-frequency (B) plots of Alnus-associated ectomycorrhizal fungal taxa sampled in mesh bags and root tips. 


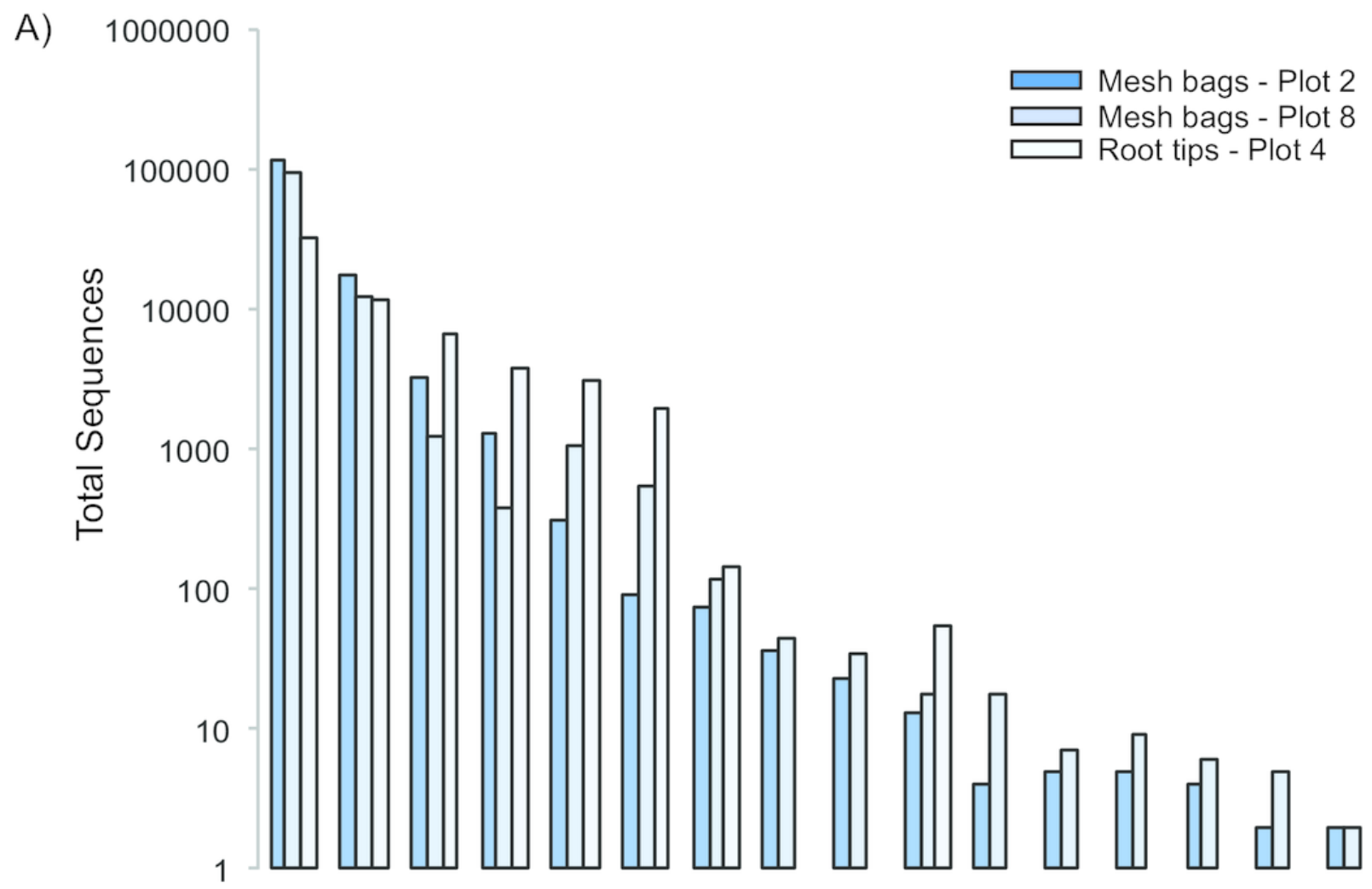

B)

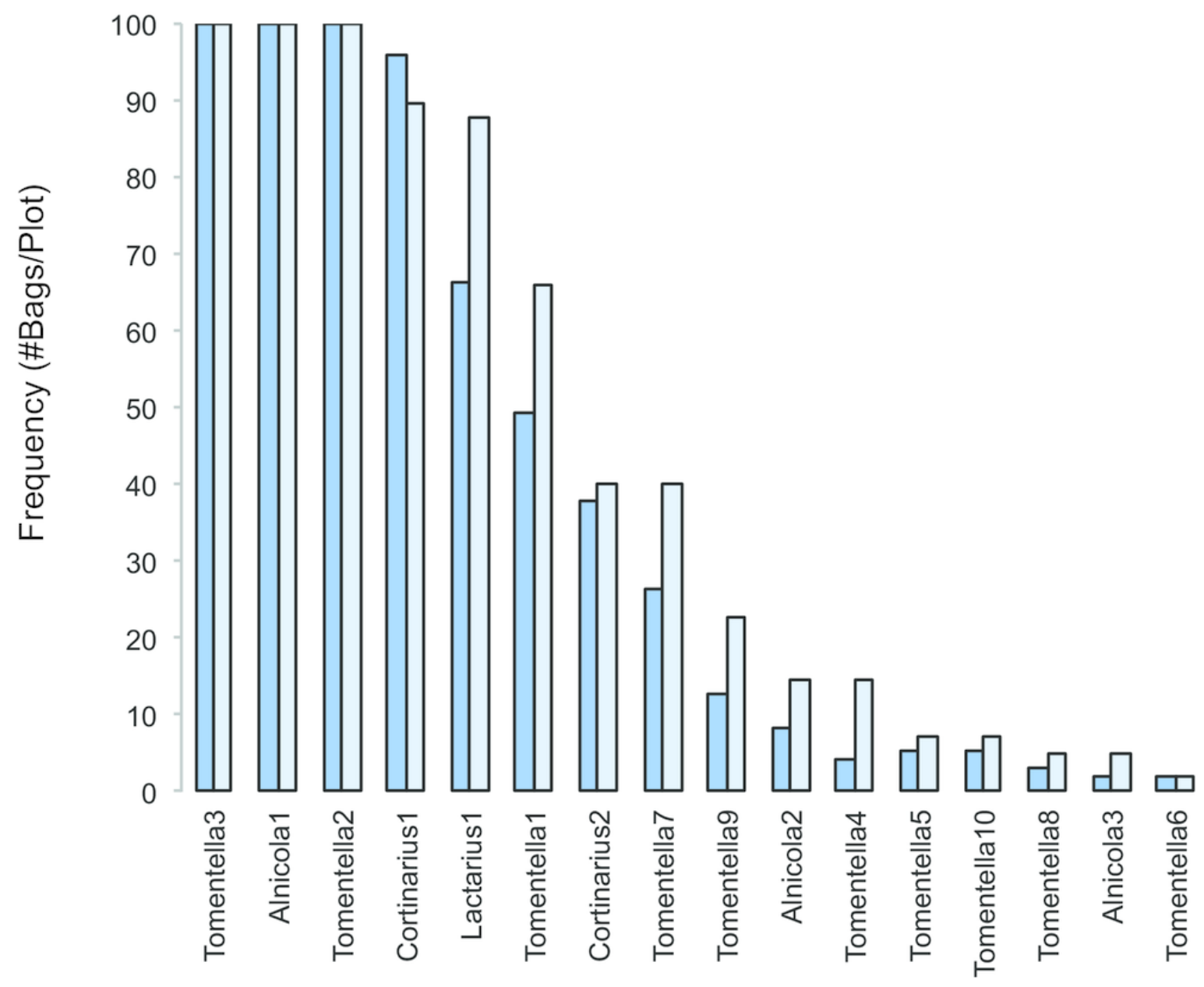




\section{Table $\mathbf{1}$ (on next page)}

C-score taxon occurrence analyses of ECM fungal communities in Plots 2 and 8.

See methods for details about datasets and null matrix type definitions. SES = Standardized Effect Size. 
Table 1. C-score taxon occurrence analyses of ECM fungal communities in Plots 2 and 8 . See methods for details about datasets and null matrix type definitions. SES = Standardized Effect Size.

\begin{tabular}{|c|c|c|c|c|c|c|}
\hline $\begin{array}{l}\text { DataSet } \\
\text { All }\end{array}$ & $\begin{array}{l}\text { Plot } \\
2\end{array}$ & $\begin{array}{l}\text { Null Matrix Type } \\
\text { Fixed-Fixed } \\
\text { Fixed-Equiprobable }\end{array}$ & $\begin{array}{r}\text { C observed } \\
173.2\end{array}$ & $\begin{array}{r}\text { C expected } \\
173.8 \\
188.1\end{array}$ & $\begin{array}{r}P \text { value } \\
0.00 \\
0.00\end{array}$ & $\begin{array}{l}\text { SES } \\
-3.35 \\
-21.2\end{array}$ \\
\hline All & 8 & $\begin{array}{l}\text { Fixed-Fixed } \\
\text { Fixed-Equiprobable }\end{array}$ & 164.4 & $\begin{array}{l}164.7 \\
172.1\end{array}$ & $\begin{array}{l}0.73 \\
0.00\end{array}$ & $\begin{array}{l}-0.75 \\
-11.8\end{array}$ \\
\hline Alnus & 2 & $\begin{array}{l}\text { Fixed-Fixed } \\
\text { Fixed-Equiprobable }\end{array}$ & 93.5 & $\begin{array}{r}92.5 \\
106.7\end{array}$ & $\begin{array}{l}0.21 \\
0.04\end{array}$ & $\begin{array}{r}0.76 \\
-1.75\end{array}$ \\
\hline Alnus & 8 & $\begin{array}{l}\text { Fixed-Fixed } \\
\text { Fixed-Equiprobable }\end{array}$ & 103.1 & $\begin{array}{l}103.2 \\
114.5\end{array}$ & $\begin{array}{l}0.47 \\
0.04\end{array}$ & $\begin{array}{l}-0.07 \\
-1.82\end{array}$ \\
\hline AlnusRootOnly & 2 & $\begin{array}{l}\text { Fixed-Fixed } \\
\text { Fixed-Equiprobable }\end{array}$ & 77.4 & $\begin{array}{l}76.7 \\
82.5\end{array}$ & $\begin{array}{l}0.74 \\
0.27\end{array}$ & $\begin{array}{r}0.54 \\
-0.59\end{array}$ \\
\hline AlnusRootOnly & 8 & $\begin{array}{l}\text { Fixed-Fixed } \\
\text { Fixed-Equiprobable }\end{array}$ & 61.2 & $\begin{array}{l}61.6 \\
78.4\end{array}$ & $\begin{array}{l}0.45 \\
0.13\end{array}$ & $\begin{array}{l}-0.26 \\
-1.15\end{array}$ \\
\hline AlnusMinusTop3 & 2 & $\begin{array}{l}\text { Fixed-Fixed } \\
\text { Fixed-Equiprobable }\end{array}$ & 200.3 & $\begin{array}{r}198.4 \\
228.25\end{array}$ & $\begin{array}{l}0.27 \\
0.04\end{array}$ & $\begin{array}{r}0.59 \\
-1.72\end{array}$ \\
\hline AlnusMinusTop3 & 8 & $\begin{array}{l}\text { Fixed-Fixed } \\
\text { Fixed-Equiprobable }\end{array}$ & 178.7 & $\begin{array}{l}179.3 \\
198.6\end{array}$ & $\begin{array}{l}0.47 \\
0.03\end{array}$ & $\begin{array}{l}-0.20 \\
-1.88\end{array}$ \\
\hline AlnusTomentellaOnly & 2 & $\begin{array}{l}\text { Fixed-Fixed } \\
\text { Fixed-Equiprobable }\end{array}$ & 61.6 & $\begin{array}{l}62.6 \\
88.7\end{array}$ & $\begin{array}{l}0.77 \\
0.02\end{array}$ & $\begin{array}{l}-0.55 \\
-1.99\end{array}$ \\
\hline AlnusTomentellaOnly & 8 & $\begin{array}{l}\text { Fixed-Fixed } \\
\text { Fixed-Equiprobable }\end{array}$ & 108.3 & $\begin{array}{l}107.6 \\
109.1\end{array}$ & $\begin{array}{l}0.64 \\
0.47\end{array}$ & $\begin{array}{r}0.31 \\
-0.09\end{array}$ \\
\hline
\end{tabular}


Table 2 (on next page)

Spearman rank correlation coefficient matrices for ECM fungal communities in Plots 2 and 8.

Significant correlations are indicated in bold. Numbers over the columns of both matrices correspond to the number of the ECM fungal taxon identified in the first row. 
Table 2. Spearman rank correlation coefficient matrices for ECM fungal communities in Plots 2 and 8. Significant correlations are indicated in bold. Numbers over the columns of both matrices correspond to the number of the ECM fungal taxon identified in the first row.

$\begin{array}{lr}\text { Plot2 } & 1 \\ \text { 1. Tomentella3 } & 1.00 \\ \text { 2. Alnicola1 } & 0.00 \\ \text { 3. Tomentella2 } & -0.02 \\ \text { 4. Cortinarius1 } & -0.05 \\ \text { 5. Lactarius1 } & -0.07 \\ \text { 6. Tomentella1 } & -0.08 \\ \text { 7. Cortinarius2 } & -0.13 \\ \text { 8. Tomentella7 } & 0.16 \\ \text { 9. Tomentella9 } & 0.11 \\ \text { 10. Alnicola2 } & 0.07 \\ \text { 11. Tomentella4 } & -0.08 \\ \text { 12. Tomentella5 } & 0.15 \\ \text { 13. Tomentella10 } & 0.06 \\ \text { 14. Tomentella8 } & -0.07 \\ \text { 15. Alnicola3 } & 0.39 \\ \text { 16. Tomentella6 } & \mathbf{0 . 3 7} \\ & \\ \text { Plot8 } & 1 \\ \text { 1. Tomentella3 } & 1.00 \\ \text { 2. Alnicola1 } & 0.13 \\ \text { 3. Tomentella2 } & -0.17 \\ \text { 4. Cortinarius1 } & 0.03 \\ \text { 5. Lactarius1 } & -0.16 \\ \text { 6. Tomentella1 } & -0.14 \\ \text { 7. Cortinarius2 } & 0.06 \\ \text { 8. Tomentella7 } & -0.09 \\ \text { 9. Tomentella9 } & -0.12 \\ \text { 10. Alnicola2 } & -0.06 \\ \text { 11. Tomentella4 } & -0.05 \\ \text { 12. Tomentella5 } & -0.05 \\ \text { 13. Tomentella10 } & 0.08 \\ \text { 14. Tomentella8 } & 0.16 \\ \text { 15. Alnicola3 } & 0.28 \\ \text { 16. Tomentella6 } & -0.02 \\ & \\ \end{array}$

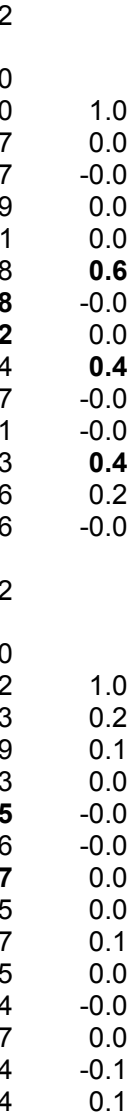

5

1.00
0.28

0.28
-0.13
-0.05
-0.03

$\begin{array}{rrr}1.00 & & \\ -0.06 & 1.00 & \\ 0.00 & 0.26 & 1.00 \\ 0.08 & -0.04 & 0.10\end{array}$

$\begin{array}{rrr}-0.05 & 0.00 & 0.26 \\ -0.03 & 0.08 & -0.04 \\ -0.05 & -0.00 & -0.02\end{array}$

-0.05
-0.05

-0.05
-0.01
-0.06

0.00
-0.04

-0.04
0.18

$\begin{array}{rr}-0.18 & 0.01 \\ -0.04 & -0.03\end{array}$

$\begin{array}{rr}0.00 & 0.08 \\ -0.03 & -0.06\end{array}$

$\begin{array}{rr}-0.03 & 0.00 \\ 0.01 & -0.03\end{array}$

$-0.04$

-0.03
-0.04

$-0.02$

$\begin{array}{rr}-0.03 & -0.10 \\ -0.06 & 0.03\end{array}$

$\begin{array}{ll}0.08 & 0.03 \\ -0.11 & 0.07\end{array}$

4

$-0.11$

$0.10 \quad 1.00$

$0.06 \quad-0.02$

$\begin{array}{ll}0.00 & 0.26 \\ 0.00 & -0.10\end{array}$

$\begin{array}{lll}-0.01 & -0.06 & -0.05 \\ -0.05 & -0.06 & -0.05\end{array}$

$\begin{array}{llll}0.01 & -0.05 & -0.06 & -0.05\end{array}$

$\begin{array}{lll}0.25 & -0.04 & 0.04\end{array}$

0.50

$0.03-0.04$

$-0.08$

8

0.03

$\begin{array}{ll}0.60 & -0.06 \\ -0.03 & 0.29\end{array}$

$-0.04$

$\begin{array}{rrrrr}-0.04 & -0.03 & 0.29 & -0.03 & -0.02 \\ -0.04 & -0.03 & -0.03 & -0.03 & -0.02\end{array}$

1.00

$-0.02-0.02$ 